\title{
Changes in Brain Tissue Oxygenation and Metabolism During Rewarming After Neonatal Encephalopathy are Related to Electrical Abnormality
}

\author{
Subhabrata Mitra ${ }^{1}$, Gemma Bale ${ }^{2}$, Isabel de Roever ${ }^{2}$, Judith Meek ${ }^{1}$, Nicola \\ J Robertson $^{1}$ and Ilias Tachtsidis ${ }^{2}$ \\ ${ }^{1}$ Institute for Women's Health, University College London and Neonatal Unit, University \\ College London Hospitals Trust, London, UK \\ ${ }^{2}$ Department of Medical Physics and Biomedical Engineering, University College London, \\ London, UK
}

\begin{abstract}
Hypoxic ischemic encephalopathy (HIE) leads to significant mortality and morbidity, and therapeutic hypothermia $(\mathrm{TH})$ has become a standard of care following HIE. After TH, the body temperature is brought back to $37^{\circ} \mathrm{C}$. Early electroencephalography (EEG) is a reliable outcome biomarker following HIE. We hypothesized that changes in cerebral oxidative metabolism, measured as $\Delta[\mathrm{oxCCO}]$, in relation to changes in brain tissue oxygenation (measured as $\Delta[\mathrm{HbD}]$ ) during rewarming will correlate with injury severity as evidenced on amplitude integrated EEG/EEG at initial presentation. Broadband near-infrared spectroscopy (NIRS) and systemic data were collected during rewarming from 14 infants following HIE over a mean period of $12.5 \mathrm{~h}$. All infants were monitored with video EEG telemetry using a standard neonatal montage. aEEG and EEG background was classified into mild, moderate and severely abnormal groups based on the background pattern. Two infants had mild, 6 infants had moderate and another 6 infants had severe abnormality at presentation. The relationship between [oxCCO] and $[\mathrm{HbD}]$ was evaluated between two groups of infants with abnormal electrical activity (mild vs moderate to severe). A significant difference was noted between the groups in the relationship between [oxCCO] and $[\mathrm{HbD}]\left(\right.$ as $\left.^{2}\right)(\mathrm{p}=0.02)$. This result indicates that the mitochondrial injury and deranged oxidative metabolism persists in the moderate to severely abnormal group during rewarming.
\end{abstract}

\section{Introduction}

Perinatal hypoxic-ischaemic (HI) brain injury causes significant morbidity and mortality. TH is beneficial following HIE and has become the standard of care in 
recent years $[1,2]$. During $\mathrm{TH}$, body temperature is lowered to $33.5^{\circ} \mathrm{C}$ and maintained over $72 \mathrm{~h}$, followed by a slow rewarming that brings body temperature back to $37^{\circ} \mathrm{C}$ over $14 \mathrm{~h}$. Rewarming is an important physiological process. Early rewarming induces neuronal apoptosis following HIE [3]. Rebound seizures have been noted during rewarming period both in an animal model [5] and neonatal intensive care [4] and further 'cooling' with slower rewarming has been beneficial. Changes in cerebral metabolism and hemodynamics have been investigated in both a preclinical model and clinical studies during and after HI, but the dynamic effects of rewarming on newborn cerebral metabolism and hemodynamics have not yet been fully investigated. We earlier reported that the relationship between mitochondrial metabolism and cerebral oxygenation becomes more impaired with rising severity of injury [5].

Using a broadband NIRS system, we monitored changes in oxidised cytochromec-oxidase $(\Delta[\mathrm{oxCCO}])$, oxy-haemoglobin $\left(\Delta\left[\mathrm{HbO}_{2}\right]\right)$ and deoxy-haemoglobin $(\Delta[\mathrm{HHb}])$ in neonatal brain in a cohort of infants following HIE [6]. Cytochromec-oxidase (CCO) is the terminal electron acceptor in the electron transport chain (ETC) and changes in its redox state indicates the state of mitochondrial oxidative metabolism and ATP synthesis [7]. Changes in total haemoglobin $\left(\mathrm{HbT}=\mathrm{HbO}_{2}+\right.$ $\mathrm{HHb})$ and haemoglobin difference $\left(\mathrm{HbD}=\mathrm{HbO}_{2}-\mathrm{HHb}\right)$ were derived. Changes in $\mathrm{HbD}$ and $\mathrm{HbT}$ reflect changes in cerebral oxygenation and changes in cerebral blood volume, respectively.

Following HIE, aEEG and EEG together are an early reliable early predictor of neurodevelopmental outcome [8].

The aim of this study was to assess the cerebral metabolic and haemodynamic changes during the rewarming period in a cohort of term infants following perinatal hypoxic-ischaemic brain injury, and compare with early injury severity assessed on aEEG and EEG. We hypothesized that the dynamic changes in cerebral oxygenation and metabolism during the rewarming period would relate to the injury severity assessed by aEEG/EEG.

\section{Methods}

This prospective observational study was approved by the research ethics committee of University College London Hospital and London Bloomsbury REC. Informed written consent was provided by parents before each study. Therapeutic hypothermia and subsequent rewarming were instituted with a servo-controlled cooling machine (Tecotherm Neo, Inspiration Healthcare, UK). Normally, the core temperature is increased by $0.5^{\circ} \mathrm{C}$ over each 2 -h period during rewarming. Rewarming data were collected from a cohort of 14 infants. Broadband NIRS monitoring was commenced as early as possible in the rewarming phase and was continued for the maximum possible period and data were collected at $1 \mathrm{~Hz}$. The optode source-detector distance of $2.5 \mathrm{~cm}$ was chosen to ensure a good depth penetration [9] with a differential path length (DPF) of 4.99 [10] which was 
corrected for the wavelength-dependency of the pathlength. The changes in chromophore concentrations were calculated from the measured changes in broadband NIR light attenuation using the modified Beer-Lambert law as applied with the UCLn algorithm [11] across 136 wavelengths (770-906nm). Systemic data were collected using ixTrend software (ixellence $\mathrm{GmbH}$, Germany) and were synchronised with the broadband NIRS data.

\section{Data Analysis}

Systemic data were down-sampled and interpolated to the NIRS data timeframe (1 $\mathrm{Hz}$ ). Artefacts from movement or changes in external lighting were removed and baseline shifts were corrected on MATLAB (Mathworks, USA). using the method suggested by Scholkmann et al. [12]. No significant difference was noted between the two sides in the broadband NIRS data and data from the left side was used for analysis. Linear regression analysis was performed to assess the relationship between $\Delta[\mathrm{oxCCO}]$ and $\Delta[\mathrm{HbD}]$ and an averaged regression coefficient $\left(\mathrm{r}^{2}\right)$ was created to compare this relationship between groups. All statistical analyses were performed using GraphPad Prism 6 (GraphPad Software, USA).

\section{Results}

NIRS and systemic data were collected over a mean period of 12.5 (5-14) h. Active cooling was started at a mean of $3 \mathrm{~h}$ of age. All infants were ventilated during rewarming. During rewarming the heart rate gradually increased from a mean of $108 / \mathrm{min}$ (range $75-130 / \mathrm{min}$ ) to $128 / \mathrm{min}(121-135 / \mathrm{min})$. The peripheral oxygen saturation $\left(\mathrm{SpO}_{2}\right)$ fell briefly at the start of rewarming, but was mostly over $95 \%$. Mean BP was stable through out the rewarming $(45-55 \mathrm{~mm} \mathrm{Hg})$. The averaged regression coefficient $\left(\mathrm{r}^{2}\right)$ between $\Delta[\mathrm{oxCCO}]$ and $\Delta[\mathrm{HbD}]$ were calculated.

All infants had some degree of abnormal electrical activity in the initial recording. Two infants had mild abnormality (normal aEEG upper $(>10 \mu \mathrm{V})$ and lower border $(>5 \mu \mathrm{V})$, but low amplitude EEG), 6 infants had moderately abnormal background (abnormal aEEG upper and/or lower margin with EEG discontinuity) and another 6 infants had severe abnormality (abnormal aEEG voltage with discontinuous or flat EEG trace) (table 1). Some of the moderate and severely abnormal traces recovered during therapeutic hypothermia. Relationships between [oxCCO] and [HbD] were evaluated between two groups of abnormal electrical activity (mild vs moderate to severe) measured in the first hour after starting active cooling. A significant difference was noted between the groups in the relationship between [oxCCO] and [HbD] (as r $\left.\mathrm{r}^{2}\right)(\mathrm{p}=0.02$, Mann-Whitney test) (Fig. 2). 


\section{Discussion}

The relationship between cerebral metabolism and oxygenation measured during rewarming following TH in a group of infants with HIE became stronger with increasing degree of brain injury, measured as aEEG/EEG abnormality at presentation. This indicates a higher oxygen dependency of the cerebral metabolism with increasing severity of injury. Availability of oxygen has a considerable influence on the oxidation of $\mathrm{CCO}$. The results imply a significant mitochondrial injury and low cerebral metabolic rate in neonatal brain following moderate to severe hypoxic ischaemic injury persists even after TH during rewarming in comparison to neonates with evidence of mild brain injury at presentation.

There was no significant pattern of changes in $\Delta[\mathrm{oxCCO}]$ with change in temperature, nor did we notice any specific cut-off temperature point which indicated any change in the trend of cerebral metabolism during rewarming.

We recently showed that the degree of injury severity was not predictable with changes in cerebral oxygenation or haemodynamics alone [13]. In near-term foetal sheep, carotid artery blood flow (CaBF) and mean arterial blood pressure (MABP) changed only transiently during rewarming. No significant difference was noted from $6 \mathrm{~h}$ onwards [3]. Peng et al. also described that, asphyxiated newborn infants had stable regional cerebral oxygenation during rewarming [14]. Our data suggest that broadband NIRS-derived measurement of cerebral oxidative metabolism can be a useful cot-side indicator of changes in mitochondrial oxidative metabolism following HIE.

Bale et al. showed that a strong relationship exists between cerebral metabolism and oxygenation during spontaneous desaturation episodes in infants with severe HIE [15]. Impairment of cerebral energy metabolism following HIE is well documented and the degree of the metabolic disturbance is related to subsequent neurodevelopmental outcome [16]. Impairment of cerebral oxidative metabolism is also evidenced with increased cerebral lactate following perinatal HI during secondary energy failure and beyond [17]. A biphasic pattern of changes in the redox state of oxCCO has been documented in both clinical [16] and preclinical studies [18]. The point of change in this biphasic pattern has been described as the point when ETC becomes significantly suppressed leading to reduction and failure of ATP production. It is likely that in severe HIE, apoptotic changes and progressive cell death impairs the mitochondrial capacity resulting in the need for a higher tissue oxygenation or increased oxygen tissue gradient [15]. In infants with mild brain injury, mitochondria are likely to have a lower oxygen saturation threshold and the oxygen tension is above the value at which their redox state becomes oxygen dependent, thus the correlation $\left(\mathrm{r}^{2}\right)$ between oxCCO and $\mathrm{HbD}$ is low.

The limitation of this study is the small number of infants enrolled, as it is not possible to generalize our findings from this study before testing it in a larger cohort. We noted that the mitochondrial metabolism-oxygenation coupling during rewarming was influenced by the severity of hypoxic ischemic brain injury. This 
has the potential to become a useful cot-side biomarker of outcome in infants with HIE.

Acknowledgments We thank all the families and neonatal staff in UCLH for their support. This project was supported by EPSRC (EP/G037256/1), The Wellcome Trust $(088429 / \mathrm{Z} / 09 / \mathrm{Z}$ and 104580/Z/14/Z) and UK Department of Health's NIHR BRC funding scheme.

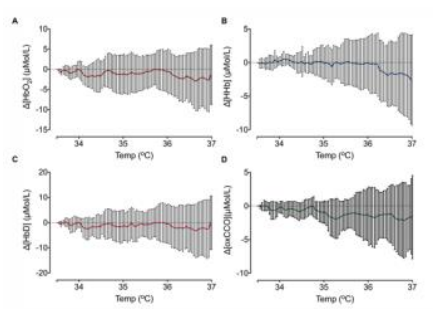

Figure. 1. Changes in $\left[\mathrm{HbO}_{2}\right](\mathbf{A}),[\mathrm{HHb}](\mathbf{B}),[\mathrm{HbD}](\mathbf{C})$ and $[\mathrm{oxCCO}](\mathbf{D})$ during rewarming period in all infants measured on the left side as mean \pm s.d. Please note that the $\mathrm{x}$-axis presents a time scale of 14 hours over which the temperature was increased from $33.5^{\circ} \mathrm{C}$ to $37^{\circ} \mathrm{C}$.

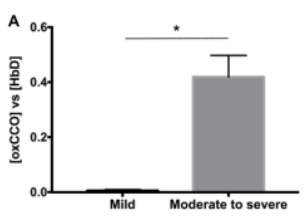

Figure 2. Relationship between [oxCCO] and $[\mathrm{HbD}]\left(\mathrm{as}^{2}\right)$ differed between the two groups of abnormal electrical activity (mild vs moderate to severe) $(\mathrm{p}=0.02)$.

\begin{tabular}{|c|l|c|}
\hline Study No & Initial electrical activity & $\begin{array}{c}{[\mathbf{o x C C O} \mathbf{~ v s}[\mathbf{H b D}]} \\
\left(\mathbf{r}^{\mathbf{2}} \mathbf{~}\right.\end{array}$ \\
\hline 1 & Severely abnormal & 0.0100 \\
\hline 2 & Severely abnormal & 0.2100 \\
\hline 3 & Mildly abnormal & 0.0018 \\
\hline 4 & Moderately abnormal & 0.7900 \\
\hline 5 & Severely abnormal & 0.0100 \\
\hline 6 & Severely abnormal & 0.6400 \\
\hline 7 & Moderately abnormal & 0.1500 \\
\hline 8 & Moderately abnormal & 0.4800 \\
\hline 9 & Severely abnormal & 0.5500 \\
\hline 10 & Moderately abnormal & 0.4300 \\
\hline 11 & Mildly abnormal & 0.0096 \\
\hline 12 & Severely abnormal & 0.3700 \\
\hline 13 & Moderately abnormal & 0.7000 \\
\hline 14 & Moderately abnormal & 0.6900 \\
\hline
\end{tabular}

Table 1. Initial aEEG/EEG characteristics and relationship between $[\mathrm{oxCCO}]$ and $[\mathrm{HbD}]\left(\mathrm{r}^{2}\right.$ values). $(n=14)$. 


\section{References}

1. NICE (2010) Therapeutic hypothermia with intracorporeal temperature monitoring for hypoxic perinatal brain injury. (http://www.nice.org.uk/guidance/ipg347/chapter/1-guidance) Committee on fetus and newborn. American Academy of Pediatrics (2014) Hypothermia and Neonatal Encephalopathy. Pediatrics 133(6): 1146-1150

2. Gerrits LC, Battin MR, Bennet L et al (2005) Epileptiform activity during rewarming from moderate cerebral hypothermia in the near-term fetal sheep. Pediatric research 57(3):342-6

3. Wang B, Armstrong JS, Lee JH et al (2015) Rewarming from therapeutic hypothermia induces cortical neuron apoptosis in a swine model of neonatal hypoxic-ischemic encephalopathy. J Cereb Blood Flow Metab 35(5):781-93

4. Kendall GS, Mathieson S, Meek J et al (2012) Recooling for rebound seizures after rewarming in neonatal encephalopathy. Pediatrics130(2):e451-5

5. Mitra S, Bale G, Meek J et al.. Relationship between cerebral oxygenation and metabolism during rewarming in newborn infantafter therapeutic hypothermia following hypoxicischemic brain injury. Adv Exp Med Biol. 2016;923:245-251

6. Bale G, Mitra S, Meek J et al (2014) A new broadband near-infrared spectroscopy system for in-vivo measurements of cerebral cytochrome-c-oxidase changes in neonatal brain injury. Biomedical optics express 5(10):3450-66

7. Richter OM, Ludwig B (2003) Cytochrome c oxidase - structure, function, and physiology of a redox-driven molecular machine. Rev. Physiol. Biochem. Pharmacol.147: 47-74

8. Murray DM, Boylan GB, Ryan CA et al (2009). Early EEG findings in hypoxic-ischemic encephalopathy predict outcomes at 2 years. Pediatrics. 2009 Sep;124(3):e459-67.

9. Grant PE, Roche-Labarbe N, Surova A et al (2009) Increased cerebral blood volume and oxygen consumption in neonatal brain injury. J. Cereb. Blood Flow Metab 29 (10):1704-13

10. Duncan A, Meek JH, Clemence M et al (1996) Measurement of cranial optical path length as a function of age using phase resolved near infrared spectroscopy. Pediatr. Res 39: 889-894.

11. Matcher S, Elwell C, and Cooper C (1995) Performance comparison of several published tissue near-infrared spectroscopy algorithms. Anal. Biochem 227,(1):54-68

12. Scholkmann F, Spichtig S, Muehlemann T et al (2010) How to detect and reduce movement artifacts in near-infrared imaging using moving standard deviation and spline interpolation. Physiol. Meas 31(5):649-62

13. Mitra S, Bale G, Highton D et al (2017). Pressure passivity of cerebral mitochondrial metabolism is associated with poor outcome following perinatal hypoxic ischemic brain injury. Cereb Blood Flow Metab. 2017 Jan 1:271678X17733639.

14. Peng S, Boudes E, Tan X et al (2015) Does Near-Infrared Spectroscopy Identify Asphyxiated Newborns at Risk of Developing Brain Injury During Hypothermia Treatment? Am J Perinatol 32(6):555-64

15. Bale G, Mitra S, de Roever I et al (2018). Oxygen dependency of mitochondrial metabolism indicates outcome of newborn brain injury. J Cereb Blood Flow Metab. 2018 May 18:271678X18777928.

16. Azzopardi D, Wyatt JS, Cady EB, et al. Prognosis of newborn infants with hypoxic-ischemic brain injury assessed by phosphorus magnetic resonance spectroscopy. Pediatr Res 1989; 25: $445-451$.

17. Hassell KJ, Ezzati M, Alonso-Alconada D et al (2015). New horizons for newborn brain protection: enhancing endogenous neuroprotection. Arch Dis Child Fetal Neonatal Ed. 2015 Nov;100(6):F541-52

18. Bainbridge A, Tachtsidis I, Faulkner SD et al (2014). Brain mitochondrial oxidative metabolism during and after cerebral hypoxia-ischemia studied by simultaneous phosphorus magnetic-resonance and broadband near-infrared spectroscopy. Neuroimage. 2014 Nov 15;102 Pt 1:173-83 